九州大学学術情報リポジトリ

Kyushu University Institutional Repository

\title{
Growth of horizontally aligned single-walled carbon nanotubes on anisotropically etched silicon substrate
}

Orofeo, Carlo M.

Graduate School of Engineering Sciences, Kyushu University

Ago, Hiroki

Institute for Materials Chemistry and Engineering, Kyushu University | Graduate School of Engineering Sciences, Kyushu University

Ikuta, Tatsuya

Graduate School of Engineering, Kyushu University

Takahasi, Koji

Graduate School of Engineering, Kyushu University

他

http://hdl. hand le. net/2324/26051

出版情報: Nanoscale. 2 (9)，pp.1708-1714，2010-09. Royal Society of Chemistry バージョン:

権利関係: (C) The Royal Society of Chemistry 2010 


\title{
Growth of Horizontally Aligned Single-Walled Carbon Nanotubes on Anisotropically Etched Silicon Substrate
}

\author{
Carlo M. Orofeo, ${ }^{\dagger}$ Hiroki Ago, ${ }^{*,+, \&}$ Tatsuya Ikuta, ${ }^{\perp}$ Koji Takahasi, $^{\perp}$ and Masaharu Tsuji ${ }^{\dagger, *}$
}

\begin{abstract}
Directional controllability of single-walled carbon nanotubes (SWNTs) is an important issue for future nanoelectronics applications. For direct integration of carbon nanotubes with modern electronics, aligned growth of carbon nanotubes on $\mathrm{SiO}_{2} / \mathrm{Si}$ is desirable. We developed a new method to horizontally align SWNTs directly on $\mathrm{SiO}_{2} / \mathrm{Si}$ substrate by creating trenches on $\mathrm{Si}(100)$ through anisotropic etching. The V-shaped trenches highly improved the alignment of SWNTs and the degree of alignment is comparable to the step-templated alignment of carbon nanotubes on crystals. The trenches also improved the density of aligned nanotubes due to the combination of "trench-guided" and gas-flow guided alignment. Our new insights on carbon nanotube alignment on $\mathrm{SiO}_{2} / \mathrm{Si}$ will greatly contribute to future large-scale nanoelectronic applications.
\end{abstract}

\section{Introduction}

Single-walled carbon nanotubes (SWNTs) have been considered as one of the promising materials for future technologies due to its exceptional mechanical and electrical 5 properties. ${ }^{1}$ For applications in nanoelectronics, the capability to control both position and direction of SWNTs is of utmost importance for the integration of SWNT to the current silicon-based electronics. This is because siliconbased electronics are configured in a highly ordered geometry. $10 \mathrm{Also}$, the nanoscale diameter of a SWNT is ideal for further integration or miniaturization of devices. Several studies have been made to characterize the device performance of a network comprising from random and overlapping nanotubes by comparing with an array of horizontally-aligned 15 nanotubes. ${ }^{2-4}$ These studies suggested that tube-tube contacts within the nanotube array greatly affect the overall device performance. For instance, it was recently reported that the junctions of the intertwined SWNT increase the resistance and that the junctions control the overall network performance. ${ }^{4}$

20 Moreover, direct growth of aligned and isolated SWNTs on silicon substrate with an oxide layer $\left(\mathrm{SiO}_{2} / \mathrm{Si}\right)$ using conventional chemical vapor deposition (CVD) methods is desirable for direct integration into the current electronic devices. However, due to the amorphous nature of $\mathrm{SiO}_{2}$, $25 \mathrm{SWNTs}$ grown on $\mathrm{SiO}_{2} / \mathrm{Si}$ are randomly oriented and overlap each other, ${ }^{5-6}$ unlike in crystal substrates where horizontal alignment is self-assembled. ${ }^{7-14}$ This led to several researches specifically aimed on the alignment of SWNTs on $\mathrm{SiO}_{2} / \mathrm{Si}^{15}$ -

30 Successful alignment of SWNTs on $\mathrm{SiO}_{2} / \mathrm{Si}$ was achieved by either controlling the CVD condition or modification of the substrate. A widely used example in the former case is the gas-flow directed alignment. ${ }^{16-20}$ It was suggested in this method that the SWNT aligns along a laminar flow of gas 35 used during CVD growth. This method assigns the "kiteflying" mechanism as responsible for the alignment. ${ }^{17}$ The mechanism suggests that during growth, the catalyst is located at the SWNT tip while floating on top of the substrate (tipgrowth) thereby avoiding contact with the substrate and thus, 40 consequently providing an argument to the long nature (up to several centimeters) of the SWNTs produced. However, the argument of producing a long SWNT with the catalyst remaining on the surface (base-growth) was also being raised by other group. ${ }^{20}$ Electric field-induced alignment during ${ }_{45} \mathrm{CVD}$ growth was also reported, ${ }^{15}$ but the degree of alignment is insufficient and it requires electrical wiring that works at high CVD temperature.

Recently, we have demonstrated alignment of SWNT based on the second approach. Our method requires modification of 50 the $\mathrm{SiO}_{2} / \mathrm{Si}$ substrate to create trenches and guide the growth of SWNTs. ${ }^{24}$ We have proposed that SWNTs were caught at the edges of the reactive ion etched (RIE) square-shaped trenches, thus aligning the nanotubes along the edge. However, SWNTs escaped due to the inconsistent shape of the 55 trench giving a lesser degree of alignment compared to the gas-flow directed alignment. ${ }^{24,25}$ Our previous results suggested that trench structure is essential for improving the degree of SWNT alignment.

In this paper, we improved the trench structure by using 60 anisotropic etching of Si wafers to produce $\mathrm{V}$-shaped trenches, which uses the different etching rates of $\mathrm{Si}\{111\}$ and $\mathrm{Si}\{100\}$ surfaces against potassium hydroxide $(\mathrm{KOH})$ etching solution. The trench shape is expected to be more defined in terms of structure than the previous RIE etched trenches. We have ${ }_{65}$ created V-shaped trenches with depths between $150 \mathrm{~nm}$ and $300 \mathrm{~nm}$, in which gas flow on trenches appeared to affect the growth and alignment of SWNTs. Anisotropic etching of Si substrates is widely used for microelectromechanical system (MEMS), but relatively large-scale structures with scale over $701 \mu \mathrm{m}$ are fabricated. ${ }^{26}$ Here, we developed a new method to create a several $100 \mathrm{~nm}$-scale patterns for the manipulation of SWNT growth.

By combining both the proposed alignment mechanisms, this paper presents a new insight of aligning SWNTs with 75 higher density and higher degree of alignment compared to 




Scheme 1. Schematic diagram showing the method to grow horizontally aligned SWNTs on patterned silicon. To create the pattern, anisotropic etching of $\mathrm{Si}(100)$ was done by carefully aligning an $\mathrm{SiO}_{2}$ mask parallel or perpendicular to $\mathrm{Si}<110>$ prior to anisotropic etching (see text for details). The mask was defined by EB lithography.

the previously reported results. ${ }^{16-20,24,25}$ Moreover, we will show that both gas-flow assisted and "trench-assisted" alignment contributed to the improvement of density and degree of alignment. Lastly, parameters like temperature, gas 5 flow rate, gas flow direction, and trench widths were varied in order to investigate scalability. The possible mechanism for alignment will be discussed based on these data.

\section{Experimental}

\section{Anisotropic Etching of $\mathrm{Si}(100)$}

10 A schematic diagram of the process is shown in Scheme 1. Wafers of $\mathrm{Si}(100)$ with a $300 \mathrm{~nm}$ surface oxide layer were purchased from SUMCO Corp. For this work, $\mathrm{SiO}_{2}$ was used as a mask as anisotropic etching requires a hard mask. The $300-\mathrm{nm}$ oxide layer was etched to $\sim 100 \mathrm{~nm}$ by soaking the 15 substrate under buffered hydrofluoric acid (BHF) solution at room temperature in order to make the oxide thin enough for better control during RIE. Spin coating of positive electron beam (EB) resist (ZEP520A, Zeon Corp.) and carefully aligning the substrate's pattern parallel or perpendicular to the $\left.{ }_{20}<110\right\rangle$ direction prior to EB lithography created a mask pattern (Scheme 1a). The position of the mask pattern is critical to the formation of $\mathrm{V}$-shaped trenches since the $\{111\}$ planes intersect at a specific crystallographic direction that gives a square-based pyramid pit shape on $\operatorname{Si}(100) .{ }^{26,27}$ The 25 different $\mathrm{SiO}_{2}$ line pattern openings of $200 \mathrm{~nm}-500 \mathrm{~nm}$ (with constant spacing of $500 \mathrm{~nm}$ ) and up to $1 \mathrm{~mm}$ in length exposed after developing (ZED-N50) and rinsing (ZMD-B), was then subjected to RIE (SAMCO 10iP) using $\mathrm{CF}_{4}$ gas to remove the $\mathrm{SiO}_{2}$ and expose the silicon surface which was not 30 covered with the EB resist (Scheme 1b).

Orientation-dependent etching was done by soaking the $\mathrm{SiO}_{2}$-masked silicon into $\mathrm{KOH}$ solution of DI water $(30 \mathrm{wt} \%)$ at $80{ }^{\circ} \mathrm{C}$ for $1 \mathrm{~min}$. It is known that (100)-oriented surfaces etch 100 times faster than (111)-oriented surfaces on

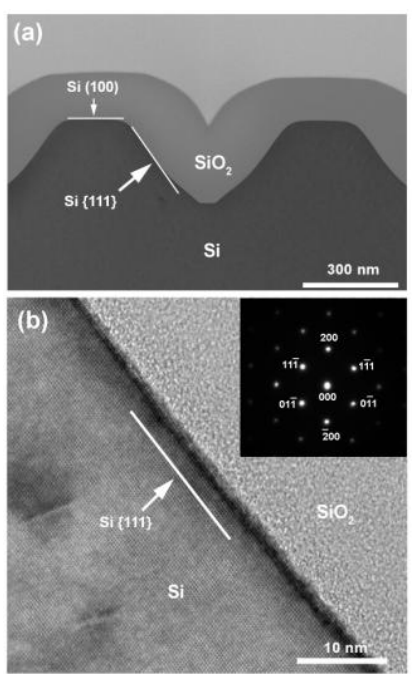

Figure 1. STEM (a) and TEM (b) images of the created trenches on silicon surface. Both images were viewed from $\mathrm{Si}\{110\}$ direction. Inset of (b) is a diffraction pattern image obtained from the Si matrix.

${ }_{35} \mathrm{KOH}^{26,27}$ With proper mask orientation, a precise V-shaped groove can be formed with $\mathrm{Si}\{111\}$ surfaces making an angle of $54.7^{\circ}$ with respect to $\mathrm{Si}(100)$. After etching, the $\mathrm{SiO}_{2}$ mask was completely removed by soaking in BHF for $3 \mathrm{~min}$ (Scheme 1d). The etched silicon was annealed under Ar gas 40 at $900{ }^{\circ} \mathrm{C}$ for 3 hours $(100 \mathrm{sccm})$, prior to thermal oxide growth, to sharpen the $\mathrm{V}$-shaped trenches. Then, the substrate surface was oxidized by heating the substrate at $900{ }^{\circ} \mathrm{C}$ while flowing oxygen $(200 \mathrm{sccm})$ bubbled through heated $\left(95{ }^{\circ} \mathrm{C}\right)$ DI water (Scheme 1e). The thickness of $\mathrm{SiO}_{2}$ can be 45 controlled by exposure time which in our case is equivalent to $\sim 200 \mathrm{~nm}$.

\section{CVD Growth of SWNTs on Trenched Silicon}

The stripe pattern of Fe catalyst was made by radio frequency (RF) magnetron sputtering (Shibaura Mechatronics Corp., ${ }_{50}$ CFS-4ES) on a $10-\mu \mathrm{m}$ photoresist openings spaced at $100 \mu \mathrm{m}$ that was previously patterned and positioned perpendicular to the substrate's trenches. The thickness of the catalyst was controlled to $<1.0 \mathrm{~nm}$ that gave the highest nanotube yield. After lift-off with acetone, the substrate was subjected to 55 alcohol CVD (Scheme 1f). After reaching $900{ }^{\circ} \mathrm{C}$ in an $\mathrm{Ar}$ flow, a $80 \mathrm{sccm}$ flow of $\mathrm{H}_{2}$ was introduced for 5 min to reduce the Fe catalyst, followed by nanotube growth with a flow of $\mathrm{H}_{2}(80 \mathrm{sccm})$ and $\mathrm{Ar}(300 \mathrm{sccm})$ bubbled through ethyl alcohol $(\mathrm{EtOH})$ that was chilled at $0{ }^{\circ} \mathrm{C}$. Geometrical ${ }_{60}$ structures of nanotubes and trenches were observed with a scanning electron microscope (SEM, HITACHI S-4800), a scanning transmission electron microscope (STEM, HITACHI HD 2300), and a transmission electron microscope (TEM, HITACHI H-9500). Atomic force microscope (AFM, Veeco ${ }_{65}$ Nanoscope IIIa) was used to profile the trenches and Raman maps were generated using micro-Raman spectrometer (JASCO NRS-2100) equipped with Ar ion laser (514.5 nm) directed onto the substrate at $\sim 1 \mu \mathrm{m}$ spot size. 



Figure 2. (a) SEM image of aligned nanotubes on trenched silicon. (b) Large scale image of the aligned nanotube arrays. The white horizontal stripes are the catalyst patterns. (c) High magnification SEM image of one of the aligned nanotubes on the trench. Indicated are the nanotubes and the trenches. Top refers to the top of the trench and bottom refers to the bottom of the trench. In this image, the nano tube follows the bottom of the trench. (d) G-band intensity map of the aligned nanotube. A long nanotube with total length of $>\sim 100 \mu \mathrm{m}$ is indicated by an arrow. (e) Raman spectrum on the aligned SWNT shown in (c).

\section{Results and Discussion}

Figure 1a shows a STEM image of our trench profile created on Si substrate. Two different crystalline planes can be seen at the $\mathrm{Si}$ interface, original $\mathrm{Si}(100)$ plane and $\mathrm{Si}\{111\}$ plane 5 that appeared after anisotropic etching. $\mathrm{SiO}_{2}$ layer which was formed after anisotropic $\mathrm{Si}$ etching fully covered the $\mathrm{Si}$ surface. It is noted that the original Si surface has relatively smooth $\mathrm{V}$-groove but the $\mathrm{SiO}_{2}$ layer gave sharp bottom edges. Shown in Figure $1 \mathrm{~b}$ is a close up TEM image of the sidewall 10 of the V-shaped groove. This cross-sectional view of $\mathrm{Si}\{111\}$ surface was seen from one of the $\{110\}$ planes. The Si substrate showed a well defined fcc lattice structure while $\mathrm{SiO}_{2}$ was completely amorphous. Inset image is the diffraction pattern of $\mathrm{Si}$ substrate.

15 Figure 2a shows the SEM image of the aligned nanotubes on the trenched silicon grown from EtOH as carbon source. The carbon nanotubes were grown from the upper area where the patterned Fe-catalyst is located. It can be seen that the grown nanotubes are straight and distinct from each other.

20 The nanotubes appeared as thick white lines due to charging effect. Most of the nanotubes are $\sim 100 \mu \mathrm{m}$ in length relatively shorter than those of the gas flow directed growth. A large scale image of the substrate is shown in Figure $2 b$ which clearly shows scalability of the method. The degree of 25 alignment of the grown nanotubes is comparable to the preliminary works of aligned carbon nanotubes grown on stepped-crystal substrate. ${ }^{8,11}$ We shall be discussing later that this improvement in alignment was influenced by the presence of trenches.

30 A high resolution SEM image of the confined nanotubes is seen in Figure 2c. The trench structure is indicated and clearly dictates the alignment direction of the carbon nanotubes (indicated in the arrows). From our observations, there is no clear and constant alignment position of carbon 35 nanotubes within the trenches but generally gives directionality of the alignment. For example, the nanotube shown in Fig. 2c aligned along the bottom of the V-trench. Further discussions on the alignment will be presented later. Figure $2 \mathrm{~d}$ shows a spatial intensity of G-band $\left(\sim 1580 \mathrm{~cm}^{-1}\right)$ 40 measured from one of the aligned nanotubes which confirms that the white line represent a carbon nanotube. A long nanotube is indicted by the arrow. A sample Raman spectrum on one of the aligned nanotubes is presented in Figure 2e. A clear G-band and radial breathing mode (RBM) were observed. 45 We did not see a significant D-band peak which should appear at $1350 \mathrm{~cm}^{-1}$, indicating that the as-grown SWNT is of high quality. From the observed RBM, $\omega$ of $\sim 175 \mathrm{~cm}^{-1}$, the diameter is estimated to be $\mathrm{d}=1.4 \mathrm{~nm}$ using the expression $d=248 / \omega$ (in $\mathrm{nm}$ ), an indication that the grown nanotubes are so single-walled. In the area close to the catalyst pattern, short carbon nanotubes were found which is consistent with studies 

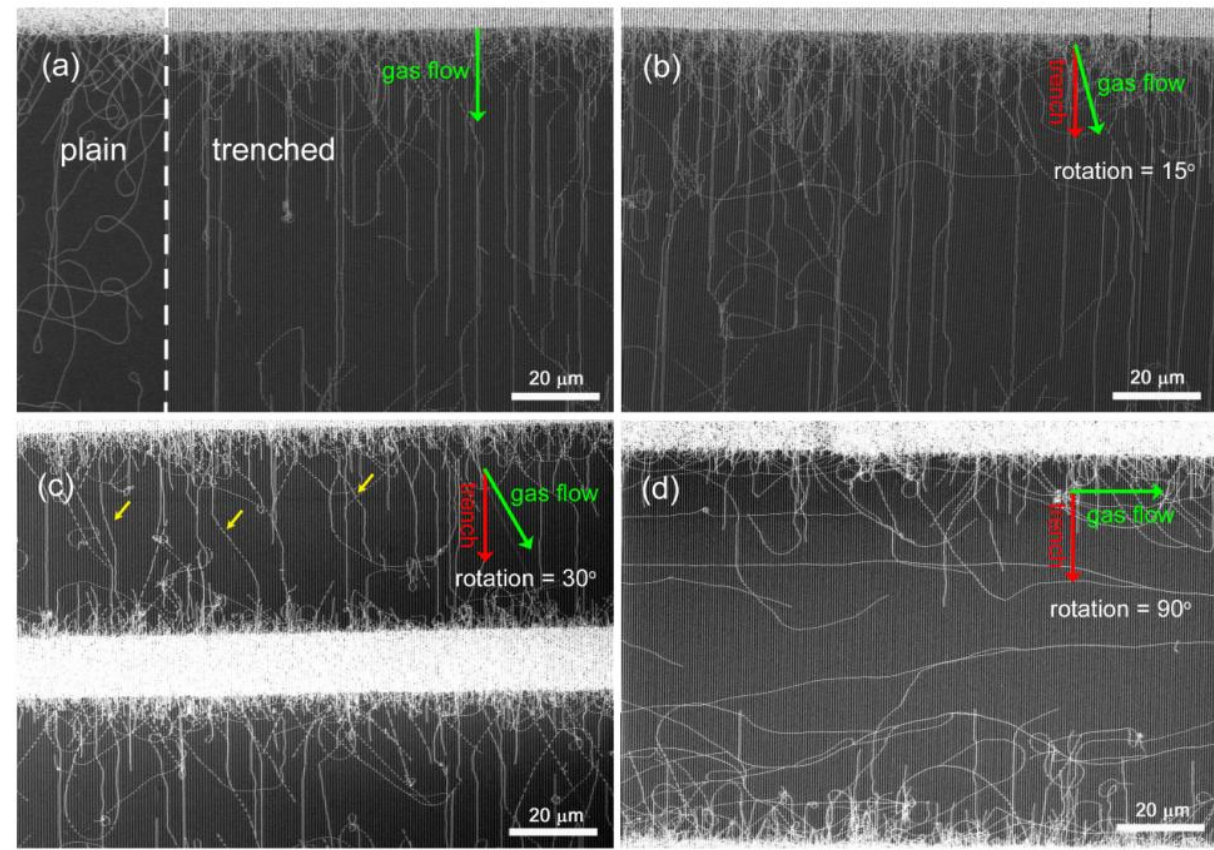

Figure 3. SEM images of aligned SWNTs on trenched Si substrates. (a) Comparison of the plain and trenched silicon substrate in terms of the alignment of SWNTs. (b-d) Influence of the rotation angle of the trenched silicon against the gas flow at different angles.

involving growth of carbon nanotubes on $\mathrm{SiO}_{2} / \mathrm{Si}$ substrate. ${ }^{16-}$ 17,22 Closer investigation of the SEM image in Figure 2a reveals that some short nanotubes align along the direction of the trenches. This leads us to investigate the nature of 5 alignment of the nanotubes for our process; i.e. if alignment is "trench-assisted" or gas-flow induced. Figure 3a contrasts the alignment of SWNTs on the boundary of the patterned (right side of the white border) and plain substrate (left side of the white border). Obvious differences in terms of alignment are 10 seen with the random nanotubes growing on the plain substrate. It is clear that the trenches greatly improved the degree of alignment.

Previous results on gas-flow directed alignment suggests that once a "kite-flying" SWNT drops onto a plain $\mathrm{SiO}_{2}$ 15 surface, the nanotube grows in random direction and soon terminates its growth. ${ }^{19}$ This observation is also possible if the growth mode is base-growth; that is the catalyst stays on the surface and the nanotubes slides. Reina et al. suggested that there is a certain critical length needed for gas-flow induced 20 alignment to occur. ${ }^{20}$ For this type of growth mechanism, we speculate that the presence of trenches prevents random sliding but instead guides the nanotubes along the trenches. Thus near the catalyst pattern, relatively short lengths $(<40$ $\mu \mathrm{m}$ ) of aligned SWNTs can be frequently found.

25 To further investigate the effect of trenches on the alignment, the trench pattern's direction was varied with respect to the gas flow direction by changing the sample orientation. This method allows the investigation of the contribution of the trenches and gas flow to the alignment of 30 carbon nanotubes. Figure $3 \mathrm{~b}-3 \mathrm{~d}$ shows the carbon nanotubes' alignment dependence on the gas flow direction with respect to the trenches. Interestingly, there was still nanotube alignment even if the substrate was rotated at $30^{\circ}$. In fact, not much difference was seen from the slightly rotated substrate $35\left(15^{\circ}\right.$, Fig. $\left.3 \mathrm{~b}\right)$. At $30^{\circ}$ rotation, similar alignment along the trenches was seen as in the un-rotated (Fig. 3a). However, some nanotubes were bent along the direction of the gas flow (indicated by yellow arrows), while some grow along the direction of the trenches unaffected by the gas flow. For the 40 latter's case, we argue that the alignment was trench-guided. At $90^{\circ}$ rotation (Figure 3d), typical gas-flow aligned SWNTs were predominant. However, short nanotubes which are aligned along the trench pattern were also present near the catalyst pattern. It is worth noting that the density of gas-flow 45 aligned carbon nanotubes in Figure $3 d$ is not as high as the unrotated or slightly rotated substrates. Therefore, the aligned nanotubes in Figure $3 b-3 c$ cannot all be contributed to the gas flow. We believe that the alignment of the nanotubes was realized by a combination of gas-flow induced and trench50 induced alignment.

Furthermore, we note that the aligned nanotubes grew in both directions from the catalyst line. Aligned carbon nanotubes that grow opposite the gas flow must be trenchinduced, because the gas-flow induced the nanotubes to grow ${ }_{55}$ downstream of the CVD reactor. Plotted in Figure $4 \mathrm{a}$ is the distribution of length of nanotubes on one of a 10-mm long catalyst line of the substrate from Figure $2 \mathrm{~b}$. This count per unit length will be the basis of comparison from other studies. As a comparison, our total density for nanotubes with length ${ }_{60} \sim 100 \mu \mathrm{m}$ at 67 nanotube/10 $\mathrm{mm}$ is roughly at least twice than the previously reported gas-flow directed results. ${ }^{16-20}$ The forward length refers to the length of the carbon nanotubes 
(a)

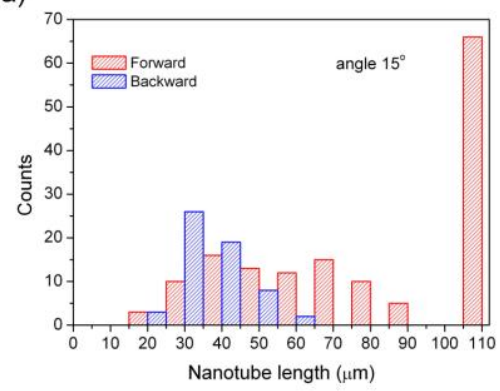

(b)

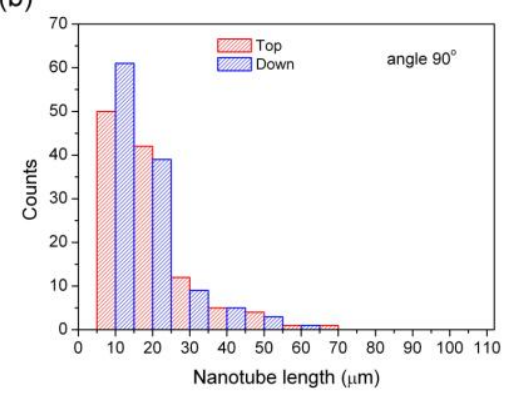

Figure 4. (a) Length distribution of the aligned SWNTs in Figure 3b. Forward and backward refers to the direction of the growth of the nanotubes with respect to the gas flow direction. (b) Length distribution of the aligned SWNTs along the trenches in Figure $3 \mathrm{~d}$ (Number of SWNTs aligned parallel to the gas flow is not indicated in the histrogram). Top and bottom refers to the direction of the nanotubes with respect to the location of the catalyst line (forward/backward is not used because the gas flow is normal to the trenches). Total length of the catalyst line by which the nanotubes were counted is $10 \mathrm{~mm}$.

that were aligned in the direction of the gas flow and the backward length is the length of the nanotubes that were aligned against the gas flow. It can be seen that the forward length (red) have two different length distributions; broad 10$590 \mu \mathrm{m}$ and a sharp $>100 \mu \mathrm{m}$. Because the distance between the catalyst lines is $100 \mu \mathrm{m}$, the long tubes with $>100 \mu \mathrm{m}$ are counted as $110 \mu \mathrm{m}$. Therefore, it can be argued that most of the forward grown nanotubes are grown via "kite-flying" mechanism where it is expected to be longer. For the trenches 10 to influence the alignment, the carbon nanotube must be sliding. The relatively smooth $\mathrm{SiO}_{2}$ surface as shown in Figure 1a allows the nanotubes to slide along the surface. Thus, the short nature of carbon nanotubes in both forward and reverse direction is expected as it must overcome the
15 frictional forces brought about by the SWNT-substrate interaction (due to the not so atomically flat substrate) during sliding.

The occurrence of both sliding and "kite-flying" mode of alignment can be greatly contrasted by rotating the substrate 20 by $90^{\circ}$ prior to CVD growth as was shown in Figure $3 \mathrm{~d}$. This allows us to distinguish the gas-flow induced and trenchguided alignment. The histogram shown in Figure $4 \mathrm{~b}$ is the number density of the nanotubes aligned along the trenches (trench-induced) of the substrate in Figure $3 \mathrm{~d}$. The top and 25 bottom indicates the position of the catalyst where the carbon nanotubes are originating (the number of gas-flow assisted alignment is not displayed). Most nanotubes were relatively short $(10-20 \mu \mathrm{m})$ and are growing from both sides of the catalyst pattern. Obviously the SWNTs shown in Fig 4 b are 30 trench-guided.

From the above results, we conclude that in order to attain highly dense and aligned nanotubes, it is necessary to keep the nanotubes sliding by extended growth durations assuming that the catalysts remain active. Growth timing studies were 35 performed and the results are shown in Figure 5. Growth timing studies can also show the differences of the two alignment modes presented here. Gas-flow aligned SWNTs are expected to be longer than the trenched-induced aligned SWNTs. Indeed in Figures 5a-5c, we see long and short 40 nanotubes. In Figure 5a, long nanotubes are seen although the growth duration was only 5 minutes. As the growth time increases, the number of relatively long nanotubes also increases. We believe that the aligned nanotubes shown in Figure $5 \mathrm{~b}$ and $5 \mathrm{c}$ are a combination of gas-flow induced and 45 trench-induced alignment. The density of the long and aligned nanotubes increased with increased growth time. So, we speculate that the synergetic effect of gas-flow and trenched-induced alignment greatly enhanced the density of the aligned SWNTs.

50 Here, we discuss the improved degree of alignment of SWNTs on our trenched silicon. As was previously shown, the gas-flow induced grown nanotubes can be aligned in the trenches even if the trench direction is slightly rotated from the gas flow direction. Two mechanisms were proposed for 55 this. First, the presence of trenches modified the local gas flow and changes gas flow direction such that it is directed along the trenches. However, the creation of desirable local gas flow is limited to certain degree $\left(\sim 30^{\circ}\right.$ rotation), as was presented previously. Different trench profiles were ${ }_{60}$ investigated to determine its effects on the alignment. Figure
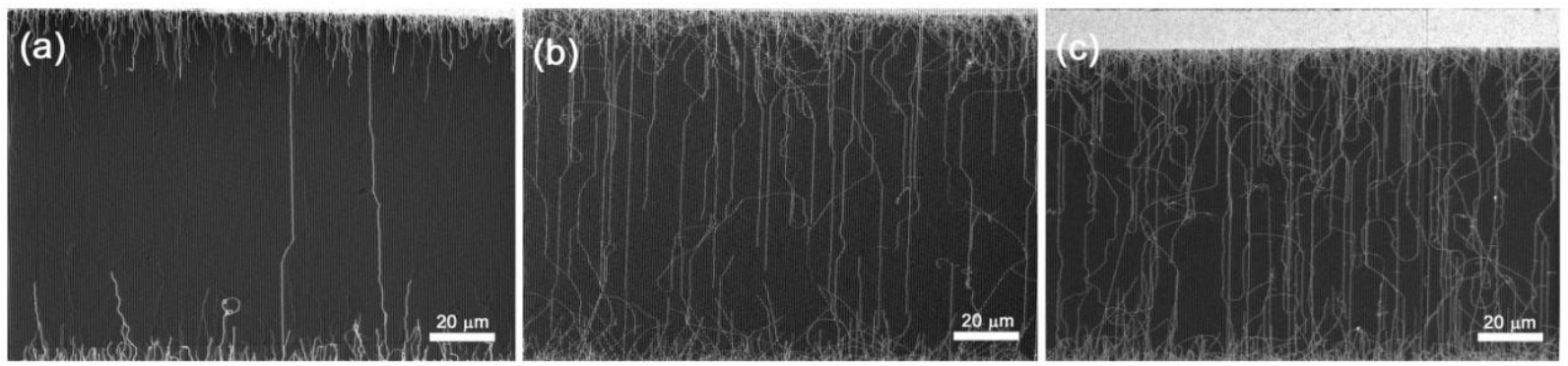

Figure 5. SEM images of the carbon nanotubes after different CVD growth times: (a) 5, (b) 30, and (c) $60 \mathrm{~min}$. 
6a shows a trench profile of wider width $(\sim 700 \mathrm{~nm})$ and was half-etched. Inset in Figure 6a is an AFM image of the trench profile. It resulted to a lesser degree of alignment compared to the V-shaped trench. This may be due to the different gas
25 nanotubes. Similarly in our case, the alignment of nanotubes occurs at the time when the length of the grown nanotubes drops at its own weight during growth and aligns its length along the trench. However, there is a limitation to the
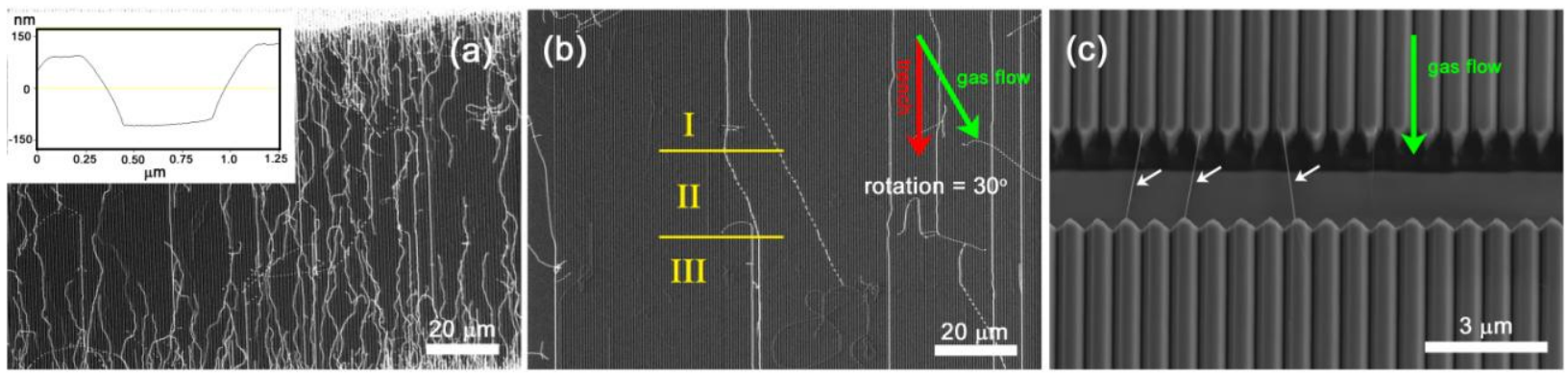

Figure 6. (a) SEM image of the aligned carbon nanotube on a squared shaped trench with a wide trench and a half-etch. Inset is the AFM profile of the trench. (b) SEM image of an aligned nanotube on a substrate that was rotated $30^{\circ}$. Straight and bent portion of the nanotube can be seen. (c) SEM image of suspended nanotubes (indicated in the arrows) in a $2-\mu \mathrm{m}$ long well.

5 flow profiles of the half-etched and V-shaped trenches. Also, the absence of V-shaped edge may not assist the trenchinduced alignment. It is noted that at different gas flow rates and $\mathrm{EtOH} / \mathrm{H}_{2}$ ratio also resulted in poor to no alignment (See Supplemental Information 1 and 2). These observations show 10 the importance of gas flow profile to the alignment of the nanotubes. Obviously, theoretical studies are needed to understand the gas flow effects on different trenches.

The other explanation to the improved degree of alignment is similar to the mechanism proposed for the formation of 15 carbon nanotubes serpentine. ${ }^{28}$ Briefly, this two-step mechanism starts with the tip-growth of a suspended nanotube along the direction of the gas flow followed by an oscillatory fall process where the nanotube adsorbs onto the surface along the step. At different gas flow direction with respect to the 20 steps, different carbon nanotube geometries including carbon nanotube serpentine can be formed. ${ }^{28}$ For stepped crystal substrates, the combined forces of surface adhesion from the substrate-carbon nanotube interaction and the aerodynamic drag caused by the gas flow induced bending of carbon
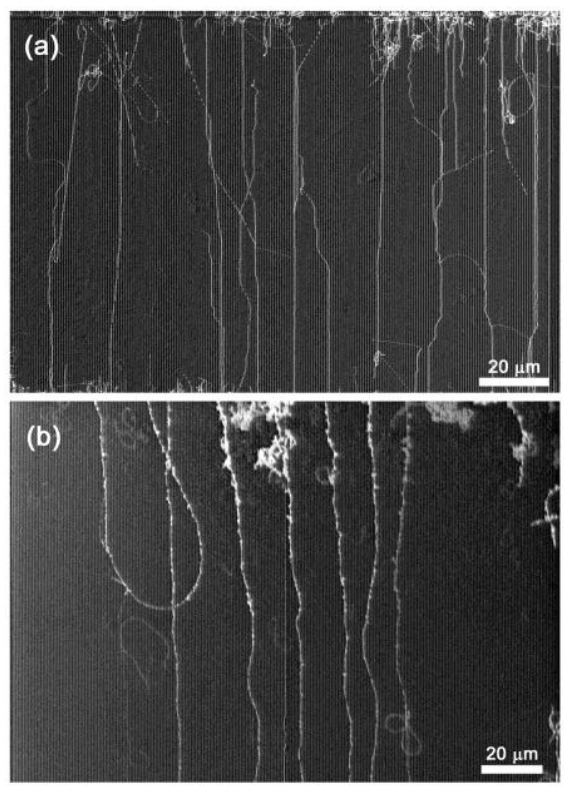

Figure 7. SEM images of the carbon nanotubes grown at different temperatures: (a) $850{ }^{\circ} \mathrm{C}$ (lower than normal) and (b) $950{ }^{\circ} \mathrm{C}$ (higher than normal) misalignment of the trenches and gas flow direction for such 30 mechanism to occur. No bending of nanotubes was seen when the substrate was rotated at $90^{\circ}$ with respect to the gas flow direction because we think that the distance between trenches is far enough that it cannot influence and induced alignment in the same manner as described previously, ${ }^{28}$ that is, the 35 aerodynamic force from the gas is the only dominant force during alignment. Figure $6 \mathrm{~b}$ shows a SEM image of the substrate with trenches rotated at $30^{\circ}$ with respect to the gas flow. Straight and aligned nanotubes aligned along the trench (shown in I), bend at Region II, and straightened again in ${ }_{40}$ Region III. Note that in Region II, the bent part was aligned with the gas flow direction which can be explained by the carbon nanotube-serpentine formation mechanism. Another proof of the mechanism is the presence of suspended nanotubes on a $2 \mu \mathrm{m}$ well-shaped barrier created between the 45 catalyst patterns as shown in Figure 6c. The suspension of the nanotubes on the well-shaped barrier clearly proves that nanotubes are grown in kite-flying mechanism. It can also be observed that it realigns in another trench after crossing the well-shaped barrier, an observation on the influence of $\mathrm{V}$ 50 shaped trenches on the alignment.

Previous studies show that temperature plays an important role in the growth of gas flow-aligned SWNT in alcohol CVD system. $^{22,29}$ These studies also suggest that there is a balance between gas flow alignment and density. Gas-flow induced 55 alignment favors high temperature but also favors formation of amorphous carbon and graphite, decreasing the density. This trade-off is interesting to see in our system since the primary goal is to achieve aligned and dense nanotubes. Figure 7 a shows a SEM image of aligned carbon nanotubes 60 grown at $850{ }^{\circ} \mathrm{C}$. The same degree of alignment was seen but the density was lower compared to nanotubes grown at $900{ }^{\circ} \mathrm{C}$, because the catalyst activity depends on CVD temperature. As temperature is increased to $950{ }^{\circ} \mathrm{C}$, we noticed that the trenches cannot influence the alignment. As shown in Figure ${ }_{65} 7 \mathrm{~b}$ the familiar gas-flow directed alignment was seen. It is also noted that at higher temperature, amorphous carbon sticking to carbon nanotubes can be observed consistent with 
alcohol CVD grown nanotubes at higher temperatures. ${ }^{21}$ We take note that the trench profile is stable at the higher CVD temperature and no noticeable changes in the profile was seen (Figure S3). This observation can have an implication to our 5 proposed alignment theory. At higher temperature, gas flow alignment is favorable and disrupts or changes the gas profile such that the trenches cannot induce alignment.

It has been accepted that gas-flow induced alignment is the major mechanism for aligning carbon nanotubes of $\mathrm{SiO}_{2} / \mathrm{Si}$ 10 substrate. Here, we present that carbon nanotubes can also be aligned with the aid of trenches. This "trench-induced" alignment is due to the confinement of carbon nanotubes within the trenches as it slides during growth. Furthermore, the presence of appropriate trench structure is necessary to

15 further improve the density and degree of alignment of nanotubes regardless of alignment mechanism.

\section{Conclusions}

We have succeeded in aligning SWNTs directly grown on $\mathrm{SiO}_{2} / \mathrm{Si}$ substrate with artificial trenches. The $\mathrm{V}$-shaped 20 trench was created by anisotropically etching $\mathrm{Si}(100)$ prior to growing SWNTs. Our grown nanotubes have a higher degree of alignment than the normal gas-flow induced aligned carbon nanotubes. Moreover, the degree of alignment was improved by the presence of trenches. The combination of gas-flow ${ }_{25}$ induced and trench-induced alignment enhanced the total SWNT density. We have proposed that the trench profile and temperature can affect the degree of alignment. It is expected that our method can greatly contribute to the integration of carbon nanotubes to the nanoelectronics where high density is 30 desirable and high controllability is a requirement.

\section{Acknowledgements}

This work was supported by the Grant-in-Aid for Scientific Research from MEXT and PRESTO-JST. CMO acknowledges the support from Global COE project for 35 partially funding this research.

\section{Notes}

${ }^{\dagger}$ Graduate School of Engineering Sciences, Kyushu University, Fukuoka 816-8580, Japan

$\$$ Institute for Materials Chemistry and Engineering, Kyushu University, 40 Fukuoka 816-8580, Japan; E-mail: ago@cm.kyushu-u.ac.jp

$\S$ PRESTO, Japan Science and Technology Agency (JST), Kawaguchi, Saitama 332-0012, Japan

${ }^{\perp}$ Graduate School of Engineering, Kyushu University, Fukuoka 8190395, Japan

$45 \dagger$ Electronic Supplementary Information (ESI) available: SEM images of SWNTs grown under different CVD conditions. See DOI: $10.1039 / \mathrm{b} 000000 \mathrm{x} /$

\section{References}

501 R. H. Baughman, A. A. Zakhidov and W. A. de Heer, Science, 2002, 297, 787.

2 C. Kocabas, N. Pimparkar, O. Yesilyurt, S.J. Kang, M. A. Alam and J. A. Rogers, Nano Lett., 2007, 7, 1195
3 S. J. Kang, C. Kocabas, T. Ozel, M. Shim, N. Pimparkar, M. A. Alam, $55 \quad$ S. V. Rotkin and J. A. Rogers, Nat. Nanotech., 2007, 2, 230.

4 P. N. Nirmalraj, P. E. Lyons, S. De, J. N. Coleman and J. J. Boland, Nano Lett., 2009, 9, 3890.

5 J. Kong, H. T. Soh, A. M. Cassell, C. F. Quate and H. Dai, Nature, 1998, 395, 878 .

606 E. S. Snow, J. P. Navak, P. M. Campbell and D. Park, Appl. Phys. Lett., 2003, 82, 2145.

7 H. Ago, K. Nakamura, K. Ikeda, N. Uehara, N. Ishigami and M. Tsuji, Chem. Phys. Lett., 2005, 408, 433.

8 A. Ismach, L. Segev, E. Wachtel, E. and Joselevich, Ang. Chem. Int. $65 E d ., 2004, \mathbf{4 3}, 6140$.

9 S. Han, X. Liu, and C. J. Zhou, J. Am. Chem. Soc., 2005, 127, 5294

10 C. Kocabas, S. H. Hur, A. Gaur, M. A. Meitl, M. Shim and J. A. Rogers, Small, 2005, 1, 1110.

11 H. Ago, N. Ishigami, R. Ohdo, K. Ikeda and M. Tsuji, Appl. Phys. 70 Lett., 2007, 90, 123112.

12 L. Ding, D. Yuan and J. Liu, J. Am. Chem. Soc. 2008, 130, 5428.

13 C. Kocabas, S. Kang, T. Ozel, M. Shim and J. A. Rogers, J. Phys. Chem. C, 2007, 111, 17879.

14 N. Ishigami, H. Ago, K. Imamoto, M. Tsuji, K. Iakoubovskii and N. 75 Minami, J. Am. Chem. Soc., 2008, 130, 9918.

15 A. Ural, Y. Li and H. Dai, Appl. Phys. Lett., 2002, 81, 3464.

16 S. Huang, X. Cai and J. Liu, J. Am. Chem. Soc., 2003, 125, 5636.

17 S. Huang, M. Woodson, R. Smalley and J. Liu, Nano Lett., 2004, 4, 1025.

8018 Z. Yu, S. Li and P. J. Burke, Chem. Mater., 2004, 16, 3414.

19 L. X. Zheng, M. J. O’Connell, S. K. Doorn, X. Z. Liao, Y. H. Zhao, E. A. Akhadov, M. A. Hoffbauer, B. J. Roop, Q. X. Jia and R. C. Dye, et al., Nat. Mater., 2004, 3, 673.

20 A. Reina, M. Hofmann, D. Zhu and J. Kong, J. Phys. Chem. C, 2007, 85 111, 7292.

21 Y. Qian, S. Huang, F. Gao, Q. Cai, L. Zhang and W. Hu, J. Phys. Chem. C, 2009, 113, 6983.

22 L. Zheng, B. C. Satishkumar, P. Gao, Q. Zhang, J. Phys. Chem. C, 2009, 113, 10896.

9023 N. Yoshihara, H. Ago, K. Imamoto, M. Tsuji, T. Ikuta and K. Takahashi, J. Phys. Chem. C, 2009, 113, 8030.

24 C. M. Orofeo, H. Ago, N. Yoshihara and M. Tsuji, Appl. Phys. Lett., 2009, 94, 53113.

25 T. Kamimura and K. Matsumoto, Appl. Phys. Express, 2009, 2, 15005 .

26 K. Bean, IEEE Transactions on Electron Devices, 1978, Ed-25, 1185 1193.

27 S. M. Sze, Semiconductor Devices: Physics and Technology in Lithography and Etching, John Wiley \& Sons, 2nd Ed: New York, 2001, pp 428-429.

28 N. Gelblinger, A. Ismach and E. Joselevich, Nat. Nanotech., 2008, 3, 195.

29 B. Zhang, G. Hong, B. Peng, J. Zhang, W. Choi, J. M. Kim, J. Choi and Z. Liu, J. Phys. Chem. C, 2009, 113, 5341. 